\title{
Cinnamon Use in Type 2 Diabetes: An Updated Systematic Review and Meta-Analysis
}

\author{
Robert W. Allen, PbarmD ${ }^{1}$ \\ Emmanuelle Scbwartzman, \\ PharmD ${ }^{1,2}$ \\ William L. Baker, PharmD, BCPS \\ $(\mathrm{AQCV})^{3,4}$ \\ Craig I. Coleman, PharmD ${ }^{3}$ \\ Olivia J. Pbung, PharmD ${ }^{1,2}$ \\ ${ }^{1}$ College of Pharmacy, Western University \\ of Health Sciences, Pomona, California \\ ${ }^{2}$ Western Diabetes Institute, Pomona, \\ California \\ ${ }^{3}$ School of Pharmacy, University of Con- \\ necticut, Storrs, Connecticut \\ ${ }^{4}$ School of Medicine, University of Con- \\ necticut, Farmington, Connecticut
}

Conflicts of interest: authors report none

\section{CORRESPONDING AUTHOR}

Olivia J. Phung, PharmD

College of Pharmacy

Western University of Health Sciences

305 E Second St

Pomona, CA 91766

ophung@westernu.edu

\begin{abstract}
PURPOSE Cinnamon has been studied in randomized controlled trials (RCTs) for its glycemic-lowering effects, but studies have been small and show conflicting results. A prior meta-analysis did not show significant results, but several RCTs have been published since then. We conducted an updated systematic review and meta-analysis of RCTs evaluating cinnamon's effect on glycemia and lipid levels.
\end{abstract}

METHODS MEDLINE, Embase, and Cochrane Central Register of Controlled Trials (CENTRAL) were searched through February 2012. Included RCTs evaluated cinnamon compared with control in patients with type 2 diabetes and reported at least one of the following: glycated hemoglobin $\left(A_{1}\right)$, fasting plasma glucose, total cholesterol, low-density lipoprotein cholesterol (LDL-C), high-density lipoprotein cholesterol (HDL-C), or triglycerides. Weighted mean differences (with $95 \%$ confidence intervals) for endpoints were calculated using random-effects models.

RESULTS In a meta-analysis of $10 \mathrm{RCTs}$ ( $\mathrm{n}=543$ patients), cinnamon doses of $120 \mathrm{mg} / \mathrm{d}$ to $6 \mathrm{~g} / \mathrm{d}$ for 4 to 18 weeks reduced levels of fasting plasma glucose $(-24.59 \mathrm{mg} / \mathrm{dL} ; 95 \% \mathrm{Cl},-40.52$ to $-8.67 \mathrm{mg} / \mathrm{dL})$, total cholesterol $(-15.60 \mathrm{mg} /$ $\mathrm{dL} ; 95 \% \mathrm{Cl},-29.76$ to $-1.44 \mathrm{mg} / \mathrm{dL})$, LDL-C $(-9.42 \mathrm{mg} / \mathrm{dL} ; 95 \% \mathrm{Cl},-17.21$ to $-1.63 \mathrm{mg} / \mathrm{dL})$, and triglycerides $(-29.59 \mathrm{mg} / \mathrm{dL} ; 95 \% \mathrm{Cl},-48.27$ to $-10.91 \mathrm{mg} /$ $\mathrm{dL})$. Cinnamon also increased levels of HDL-C $(1.66 \mathrm{mg} / \mathrm{dL} ; 95 \% \mathrm{Cl}, 1.09$ to 2.24 $\mathrm{mg} / \mathrm{dL})$. No significant effect on hemoglobin $\mathrm{A}_{\mathrm{lc}}$ levels $(-0.16 \% ; 95 \%, \mathrm{Cl}-0.39 \%$ to $0.02 \%$ ) was seen. High degrees of heterogeneity were present for all analyses except HDL-C (I ranging from $66.5 \%$ to $94.72 \%$ ).

CONCLUSIONS The consumption of cinnamon is associated with a statistically significant decrease in levels of fasting plasma glucose, total cholesterol, LDL-C, and triglyceride levels, and an increase in HDL-C levels; however, no significant effect on hemoglobin $A_{1 c}$ was found. The high degree of heterogeneity may limit the ability to apply these results to patient care, because the preferred dose and duration of therapy are unclear.

Ann Fam Med 2013;452-459. doi:10.1370/afm.1517.

\section{INTRODUCTION}

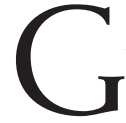
lycemic control presents a constant challenge for those with diabetes. Cardiovascular disease, one of the major complications of diabetes, is largely influenced by glycemic measures. ${ }^{1}$ To date, strategies for modifying blood glucose include pharmacologic treatment, lifestyle modification, and dietary changes. Despite an increasing body of literature focused on the use of natural supplements in the treatment of diabetes, the American Diabetes Association (ADA) does not recommend their use because clinical evidence showing efficacy is insufficient, and they lack standardized formulations. ${ }^{2}$

Cinnamon has become a natural product of interest because it has been hypothesized to provide health benefits, such as the ability to lower serum lipids and blood glucose. It has been suggested that the modality in which cinnamon expresses its effect on blood glucose can be attributed to its active component cinnamaldehyde. ${ }^{3}$ The insulinotropic effects of cinnnamaldehyde have been preliminarily investigated and are thought to 
be responsible for promoting insulin release, enhancing insulin sensitivity, increasing insulin disposal, and exerting activity in the regulation of protein-tyrosine phosphatase 1B (PTP1B) and insulin receptor kinase. ${ }^{4}$ In animal studies, aqueous cinnamon extracts have been shown to increase the expression of peroxisome proliferator-activated receptors (PPARs), which are transcriptional factors involved in the regulation of insulin resistance and adipogenesis, resulting in improved lipid and glucose metabolism. ${ }^{5}$ Several randomized controlled trials (RCTs) in humans have been conducted to explore cinnamon's efficacy; however, they report conflicting results. ${ }^{6-15} \mathrm{~A}$ meta-analysis we conducted previously did not show any statistically significant effect on glucose or lipid parameter $\mathrm{s}^{16}$; since that time, additional RCTs have been published investigating this effect. ${ }^{11-15}$ To update our prior systematic review and meta-analysis, we added the newly published evidence to determine the effect of cinnamon in patients with type 2 diabetes on glycemic and serum lipid levels.

\section{METHODS}

\section{Study Selection}

A comprehensive systematic literature search was conducted in the following databases: MEDLINE, Embase, and Cochrane Central Register of Controlled Trials (CENTRAL) from each database inception through February 2012. A search strategy was performed combining medical subject headings and text keywords for cinnamon and diabetes. No language restrictions were used. Trials were included in the analysis if they were RCTs of patients with type 2 diabetes that evaluated the use of oral cinnamon supplementation in any dose or form regardless of any additional intervention for diabetes, and that reported data on at least one of the following endpoints: glycosylated hemoglobin $\left(\mathrm{A}_{1 \mathrm{c}}\right)$ fasting plasma glucose, total cholesterol, low-density lipoprotein cholesterol (LDL-C), high-density lipoprotein cholesterol (HDL-C), or triglycerides. Two investigators reviewed potentially relevant articles independently, with differences resolved through discussion.

\section{Data Abstraction and Validity Assessment}

Two reviewers independently abstracted data from each trial using a standardized data abstraction tool. The following information was obtained from each trial: author identification, year of publication, study design, source of study funding, study duration and follow-up, study population (including study inclusion and exclusion criteria), sample size, time since diagnosis, cinnamon dose (including frequency and duration of treatment), product name/brand, formulation used, and scientific name of cinnamon species used. Baseline parameters were also collected (hemoglobin $\mathrm{A}_{1 \mathrm{c}}$ level, fasting plasma glucose level, body weight, body mass index, and total cholesterol, LDL-C, HDL$\mathrm{C}$, and triglyceride levels). Validity assessment was performed by 2 investigators independently using the Cochrane Risk of Bias Tool. ${ }^{17}$ This checklist includes 6 validity questions covering the following domains: random sequence generation, allocation concealment, blinding, blinding of outcome assessment, incomplete data reporting, and selective reporting. Each item was scored as a low, unclear, or high risk of bias.

\section{Statistical Analysis}

The mean changes in lipid parameters, serum blood glucose level, and hemoglobin $\mathrm{A}_{1 \mathrm{c}}$ level from baseline were treated as continuous variables, and the weighted mean differences (WMDs) and accompanying 95\% confidence intervals were pooled using a DerSimonian and Laird random-effects model..$^{18}$ Changes from baseline in outcomes were extracted from trials; in instances where changes were not reported directly, they were calculated from end-of-study and baseline results. Outcomes data were extracted as analyzed in that specific trial, without any additional adjustment for potential losses to follow-up. As suggested by Follmann and colleagues, ${ }^{19}$ we assumed a correlation coefficient of 0.5 between initial and final values. One trial evaluated 3 different doses of cinnamon and compared each dose with a corresponding placebo dose; results from this trial were analyzed as 3 separate trials. ${ }^{6}$

The statistical analysis was performed by using Comprehensive Meta-Analysis, version 2 (Biostat). A $P$ value of $<.05$ was considered statistically significant for all analyses. Statistical heterogeneity was assessed using the $\mathrm{I}^{2}$ statistic; $_{\text {; }}$ values of $25 \%, 50 \%$, and $75 \%$ represent low, medium, and high degrees of heterogeneity, respectively, where low levels of heterogeneity are desired.$^{20}$ To assess for the presence of publication bias, visual inspection of funnel plots was used to investigate the relationship between effect size and sample size, and Egger's weighted regression statistics tested for asymmetry. ${ }^{21}$ Subgroup analysis was performed to determine whether there was a variable effect of dosage form on the results for glycemic outcomes; RCTs evaluating cinnamon powder and RCTs evaluating cinnamon capsules were each pooled independently. Variations in the doses of cinnamon evaluated could contribute to variations in effect on glycemic parameters. We assessed whether differences in these doses affected glycemic control using random-effects meta-regression analyses.

To estimate the potential impact of recently published trials on glycemic outcomes, we conducted 
cumulative meta-analyses based on publication date whereby studies were pooled chronologically by year of publication starting with the earliest.

\section{RESULTS}

\section{Study Characteristics}

After screening for inclusion and exclusion of citations, 10 RCTs ( $\mathrm{n}=543$ patients) met all inclusion criteria (Figure 1). ${ }^{6-15}$ A number of trials required concomitant oral hyperglycemic agent therapy. ${ }^{6,7,9,11,15}$ A total of 8 trials reported usable data for hemoglobin $\mathrm{A}_{1 \mathrm{c}}$ levels ${ }^{7-12,14,15} ; 9$ trials reported results for fasting plasma glucose levels ${ }^{6-10,12-15}$; 9 trials reported results for total cholesterol levels, ${ }^{6-10,12-15}$ 8 for LDL-C levels, ${ }^{6,7,9,10,12-15} 8$ for HDL-C levels, ${ }^{7-10,12-15}$ and 9 for triglyceride levels. ${ }^{6-10,12-15}$ Patients were randomized to be treated with either cinnamon or control (dosing range $120 \mathrm{mg} / \mathrm{d}$ to $6 \mathrm{~g} / \mathrm{d}$ ) in various dosage forms (aqueous cinnamon extract or raw cinnamon powder) for a period of 4 to 18 weeks (Supplemental Table, avail-

able at http://annfammed.org/ content/11/5/452/suppl/DC1). Six trials reported evaluation of Cinnamomum cassia either as a capsule $e^{8,9,11}$ or powder. ${ }^{6,10,12}$ One trial evaluated $C$ cassia that was in a combination form with zinc gluconate and tricalcium phosphate, ${ }^{14}$ and 1 trial evaluated $C$ aromaticum, ${ }^{15}$ whereas the 2 remaining trials did not specify the type of cinnamon evaluated. ${ }^{7,13}$ All trials specified administration of cinnamon with relation to food, with 1 trial specifying cinnamon be taken before meals, ${ }^{15} 2$ trials after meals, ${ }^{6,13}$ and the remaining trials with meals. ${ }^{7-12,14}$ One trial had no losses to followup, and all patients were accounted for in the analysis ${ }^{8} ; 2$ trials specified that they conducted an intentionto-treat analysis of their data, ${ }^{10,11}$ but 1 did not report the number of patients lost to follow-up ${ }^{10} ; 2$ trials excluded the patients who withdrew from the study in their analysis ${ }^{7,15}$; and the remaining trials did not report patient disposition or whether intention-to-treat analysis was conducted. ${ }^{6,9,13,14}$ The risk of bias assessment for individual validity components is presented in Supplemental Figure 1, available at http://annfammed. org/content/11/5/452/suppl/DC1.

\section{Quantitative Data Synthesis}

Upon meta-analysis, cinnamon was associated with statistically significant reductions in levels of fasting plasma glucose (WMD = $-24.59 \mathrm{mg} / \mathrm{dL}_{;} 95 \% \mathrm{Cl},-40.52$ to $-8.67 \mathrm{mg} / \mathrm{dL}$ ), total cholesterol (WMD $=-15.60 \mathrm{mg} / \mathrm{dL}$; $95 \% \mathrm{CI},-29.76$ to $-1.44 \mathrm{mg} / \mathrm{dL}), \mathrm{LDL}-\mathrm{C}(\mathrm{WMD}=-9.42$

\section{Figure 1. Preferred reporting items in systematic reviews and meta-analyses flow diagram of study selection, inclusion, and exclusion of randomized controlled trials evaluating cinnamon on glycemic and lipid parameters.}

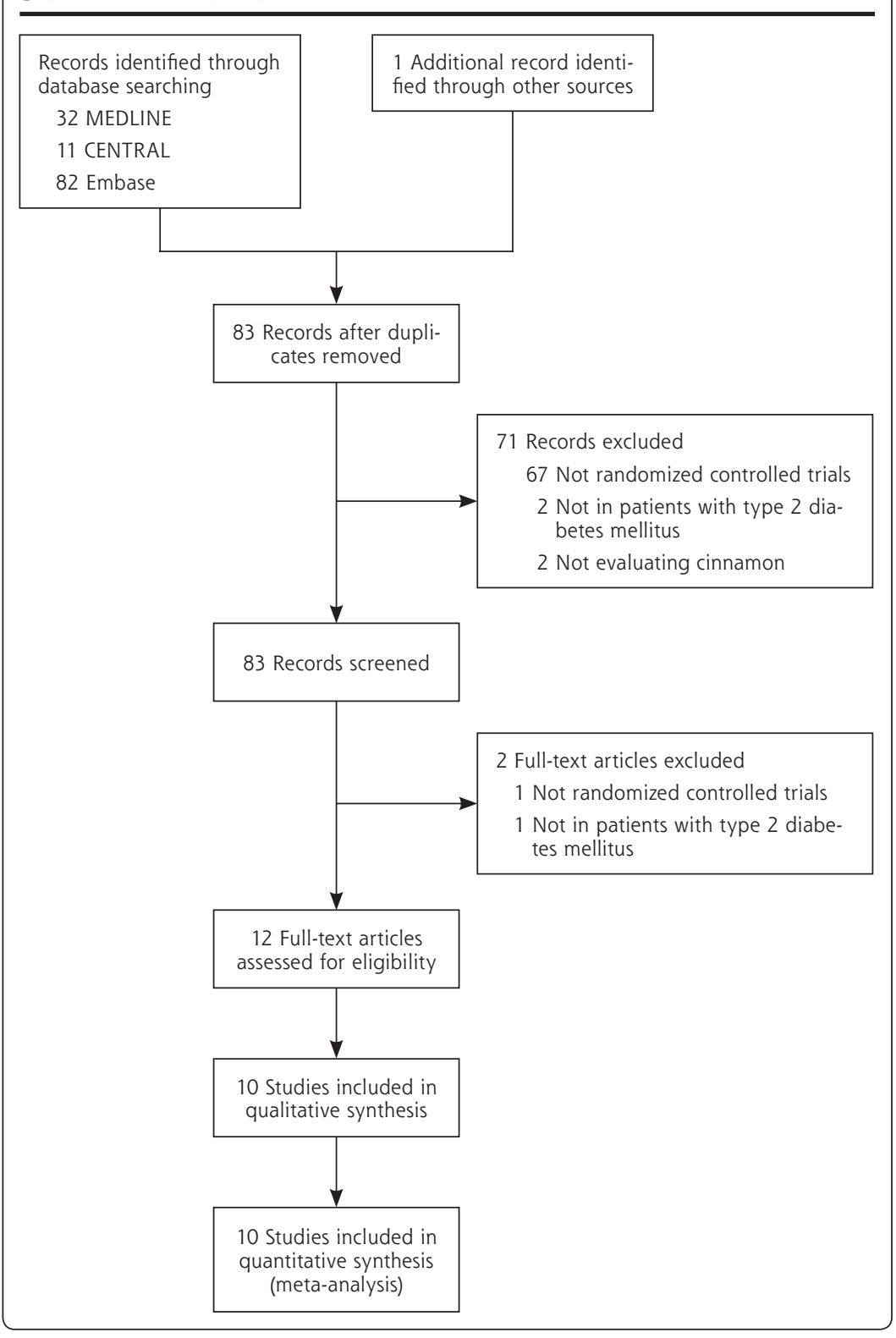




\section{Figure 2. Forest plot depicting the meta-analyses results of randomized controlled trials evaluating cinnamon on serum levels of hemoglobin $A_{1 c}$ and fasting blood glucose.}

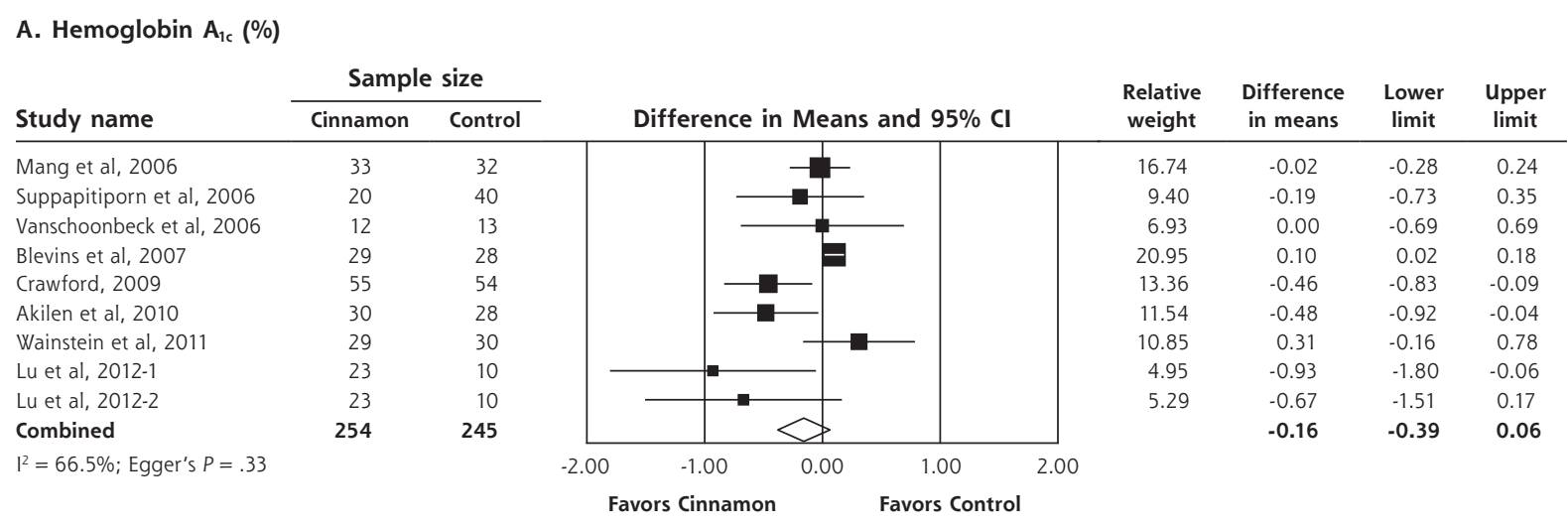

\section{B. Fasting Blood Glucose (mg/dL)}

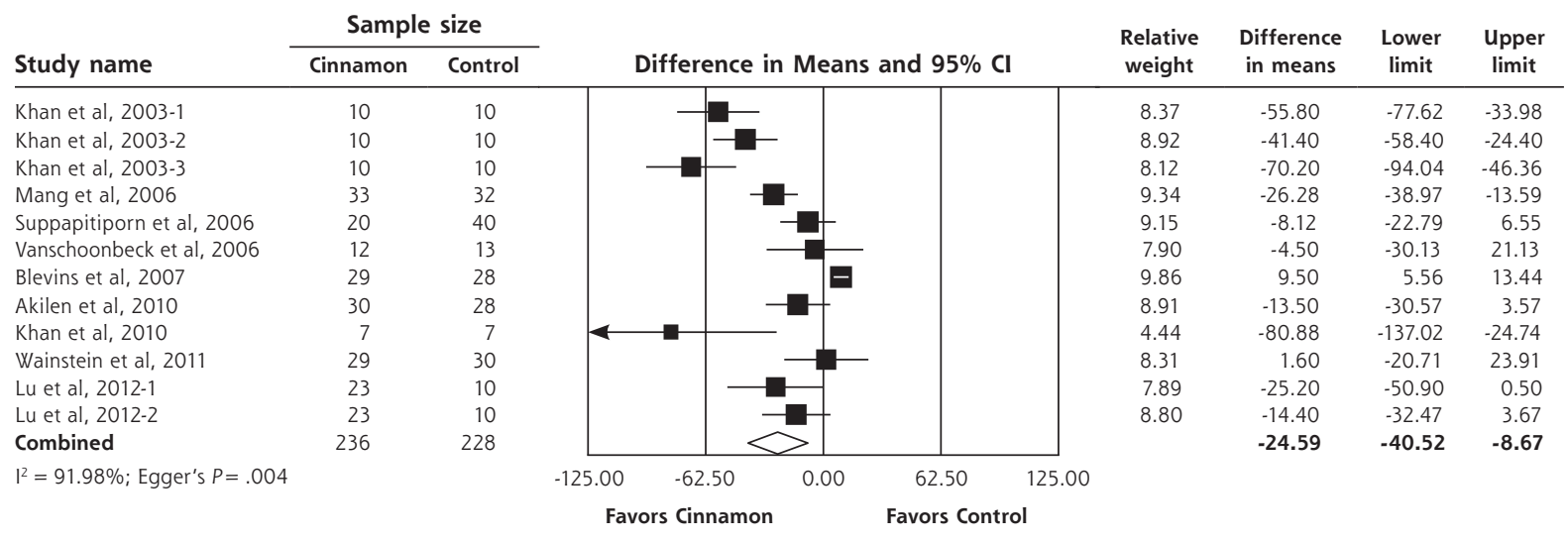

Note: Squares represent individual studies, and size of the square represents the weight given to each study in the meta-analysis. Error bars represent $95 \%$ confidence intervals. The diamonds represent the pooled results. The solid vertical line extending upward from 0.00 is the null value.

$\mathrm{mg} / \mathrm{dL} ; 95 \% \mathrm{Cl},-17.21$ to $-1.63 \mathrm{mg} / \mathrm{dL})$ and triglycerides $\left(\mathrm{WMD}=-29.59 \mathrm{mg} / \mathrm{dL}_{;} 95 \% \mathrm{CI},-48.77\right.$ to -10.91 $\mathrm{mg} / \mathrm{dL}$ ) compared with control (Figures 2 and 3). There was also a statistically significant increase in HDL-C levels (WMD $=1.66 \mathrm{mg} / \mathrm{dL}_{i} 95 \% \mathrm{CI}, 1.09$ to $2.24 \mathrm{mg} /$ $\mathrm{dL})$. There was no significant effect of cinnamon compared with control on hemoglobin $\mathrm{A}_{1 \mathrm{c}}$ (WMD $-0.16 \%$; $95 \% \mathrm{CI}_{1}-0.39 \%$ to $0.06 \%$ ) (Figure 2 ). Statistical heterogeneity was not detected in the analysis on HDL-C $\left(\mathrm{I}^{2}=0 \%\right)$, but high degrees of heterogeneity were present for hemoglobin $\mathrm{A}_{1 \mathrm{c}}$, fasting plasma glucose, triglycerides, total cholesterol, and LDL-C ( $\mathrm{I}^{2}$ ranging from $66.5 \%$ to $94.72 \%$ ). Review of funnel plots (not shown) and Egger's weighted regression $P$ values suggested potential publication bias for fasting plasma glucose analysis $(P=.004)$, but low likelihood for publication bias for hemoglobin $\mathrm{A}_{1 \mathrm{c}}$, total cholesterol, LDL-C, HDL-C and triglyceride analyses $(P>.12$ for all).

Subgroup analysis for glycemic outcomes was performed based on dosage form ${ }_{i}$ analysis on capsule forms found a significant effect on hemoglobin $\mathrm{A}_{1 \mathrm{c}}$ levels $(-0.27 \%$; $95 \% \mathrm{CI},-0.54 \%$ to $-0.01 \%)$, as well as a significant decrease in fasting glucose levels $(-16.74$ $\mathrm{mg} / \mathrm{dL}_{;} 95 \% \mathrm{CI},-25.44$ to $-8.05 \mathrm{mg} / \mathrm{dL}$ ). When evaluating powder forms, no significant benefit was seen for either hemoglobin $\mathrm{A}_{1 \mathrm{c}}(-0.01 \%$; $95 \% \mathrm{CI}, 0.37 \%$ to $0.36 \%)$ or fasting plasma glucose $\left(-27.53 \mathrm{mg} / \mathrm{dL}_{i} 95 \%\right.$ $\mathrm{CI},-55.44$ to $0.37 \mathrm{mg} / \mathrm{dL}$ ). We conducted a randomeffects meta-regression to evaluate the potential influence of cinnamon dose on hemoglobin $\mathrm{A}_{\mathrm{cc}}$ and fasting plasma glucose levels, although neither were statistically significant.

The results of the cumulative meta-analysis are presented in Supplemental Figure 2, available at http://annfammed.org/content/11/5/452/suppl/DC1.

\section{DISCUSSION}

Cinnamon has been a research interest in patients with diabetes since the 1990s, when PPARs became recog- 


\section{Figure 3. Forest plot depicting the meta-analysis results of randomized controlled trials evaluating} cinnamon on serum levels of total cholesterol, LDL cholesterol, HDL cholesterol, and triglycerides.

A. Total Cholesterol $(\mathrm{mg} / \mathrm{dL})$

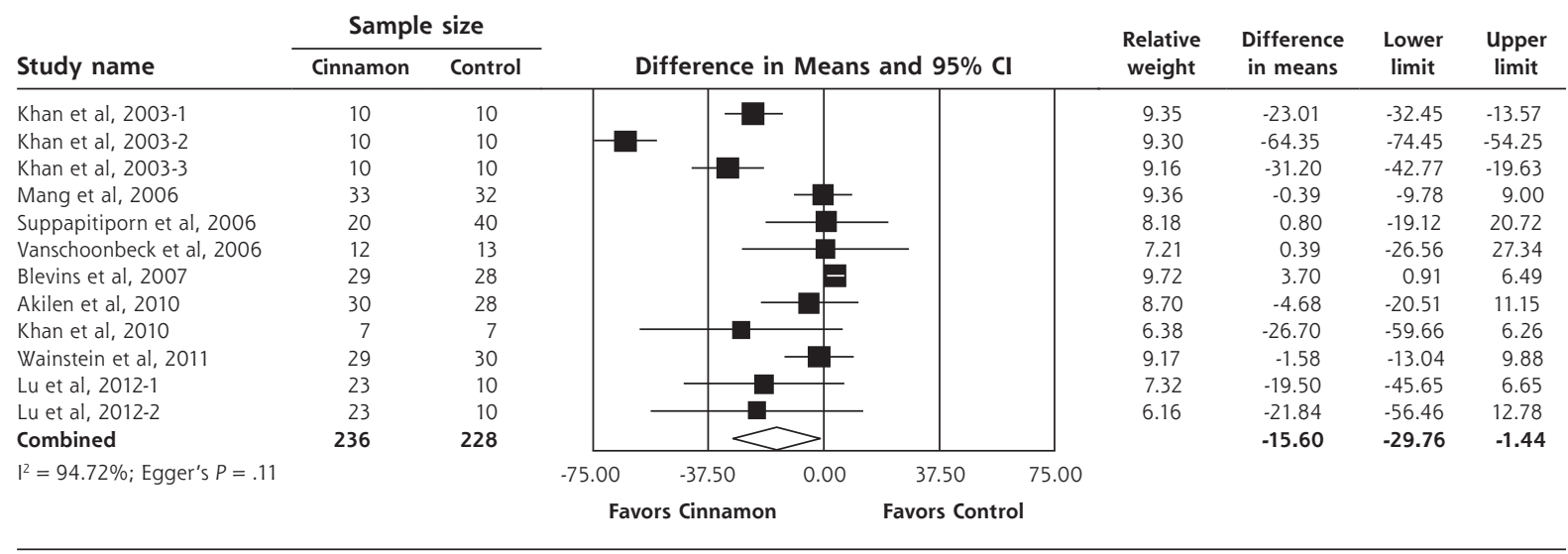

B. LDL Cholesterol (mg/dL)

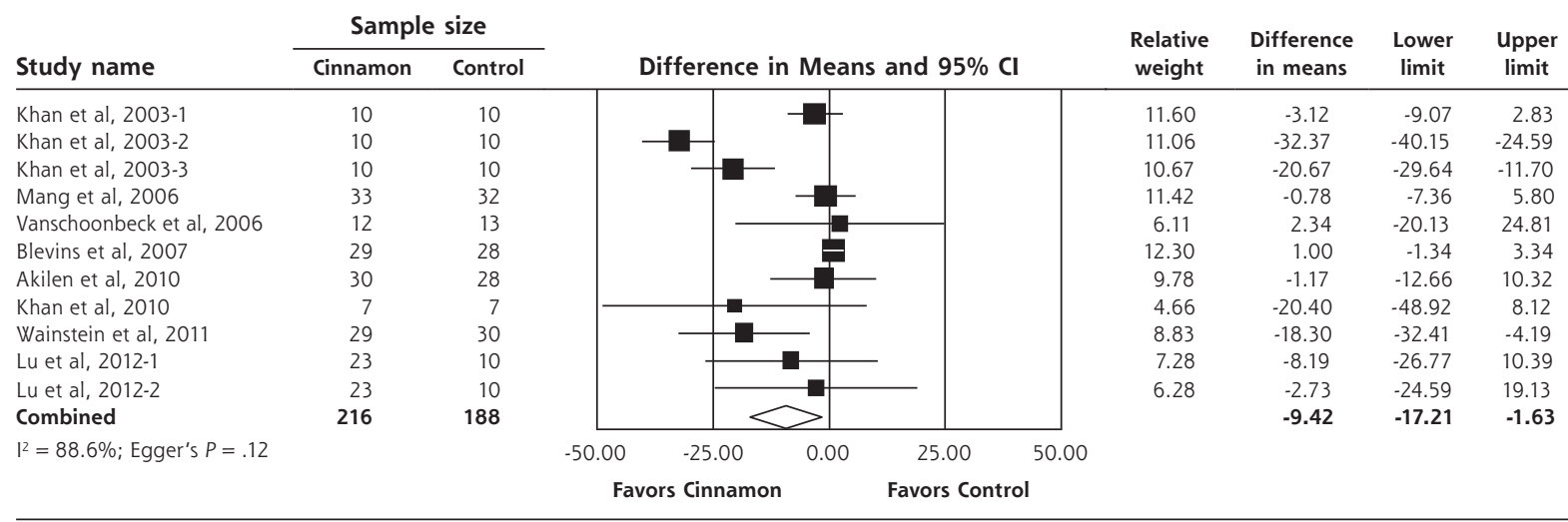

$\mathrm{HDL}=$ high-density lipoprotein; $\mathrm{LDL}=$ low-density lipoprotein.

Figure 3 continues

Note: Squares represent individual studies, and size of the square represents the weight given to each study in the meta-analysis. Error bars represent $95 \%$ confidence intervals. The diamonds represent the pooled results. The solid vertical line extending upward from 0.00 is the null value.

nized as a possible therapeutic target for dyslipidemia and diabetes. ${ }^{4}$ PPARs are ligand-activated nuclear hormone receptors expressed mainly in brown adipose tissue and the liver. Activation of various isoforms lowers levels of plasma triglycerides, elevates levels of HDL$C$, and increases insulin sensitivity. ${ }^{3}$ Glucose transport by the glucose transporters (GLUT), more specifically GLUT4, is also a primary target of diabetes research. Current research suggests that facilitating translocation through GLUT4 will increase glucose uptake and utilization, benefitting hyperglycemic control. ${ }^{5}$

Various data from RCTs show conflicting results of the effects of cinnamon on glycemic and lipid parameters. Consumption of 1 to $1.2 \mathrm{~g} / \mathrm{d}$ was associated with an increase in fasting plasma glucose levels, ${ }^{10,14}$ and hemoglobin $\mathrm{A}_{1 \mathrm{c}}$ levels ${ }^{9,10}$; however, others have reported reductions in glycemic parameters at doses between 1 to $6 \mathrm{~g} / \mathrm{d}^{6-9,12,13}$ and in hemoglobin
$\mathrm{A}_{1 \mathrm{c}} \cdot{ }^{6-9,11-13}$ The same conflict occurs in lipid parameters, at $1 \mathrm{~g} / \mathrm{d}$ total cholesterol, ${ }^{10}$ LDL-C, $^{7,12}$ and triglyceride ${ }^{10}$ levels were reported to increase, while HDL-C levels decreased. ${ }^{7-9}$ Only 1 trial identified statistically significant increases in hemoglobin $\mathrm{A}_{1 \mathrm{c}}$ and fasting plasma glucose levels; however, this trial was published only as a research brief, so many of the study characteristics were unclear. ${ }^{10}$ The trial withdrew patients if they initiated, discontinued, or adjusted any hypoglycemic or lipid-lowering medications, but the number of patients withdrawn during the 3 -month study period was not reported. ${ }^{10}$ The trial also used an intention-to-treat analysis but did not specify the data imputation method. ${ }^{10}$ It may be possible that the withdrawal of patients from the trial may bias the ability to determine cinnamon's true effect in this trial. Two trials that showed statistically significant benefit of cinnamon use on hemoglobin $A_{1 c}$ evaluated 
Figure 3. Forest plot depicting the meta-analysis results of randomized controlled trials evaluating cinnamon on serum levels of total cholesterol, LDL cholesterol, HDL cholesterol, and triglycerides (continued)

C. HDL Cholesterol (mg/dL)

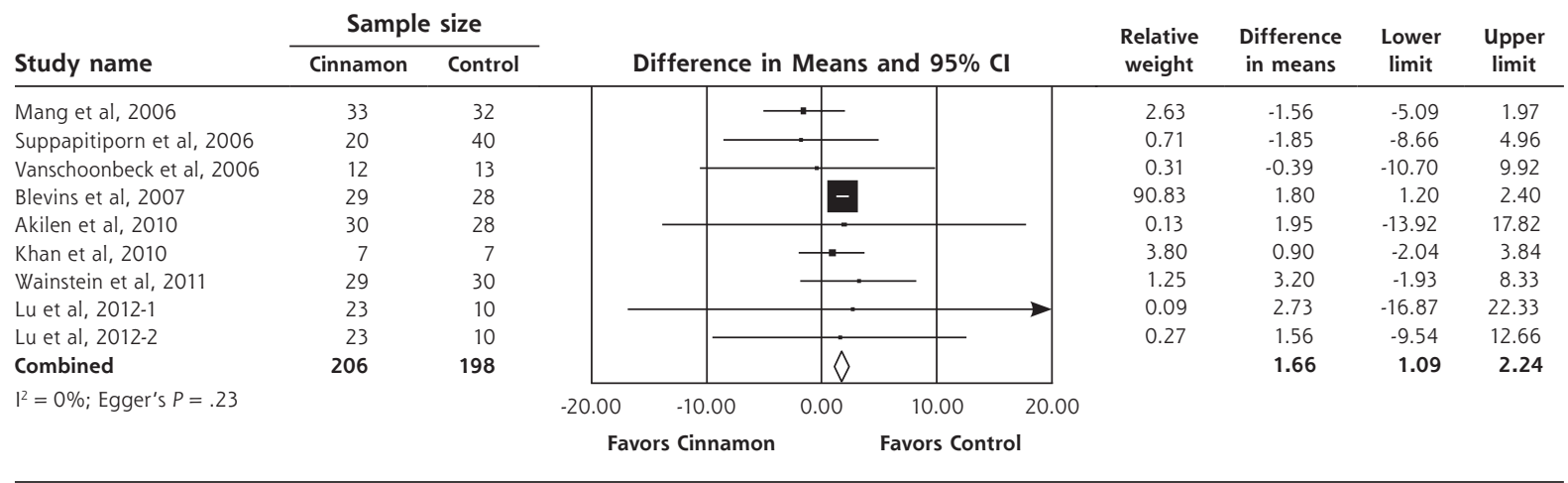

\section{Triglycerides (mg/dL)}

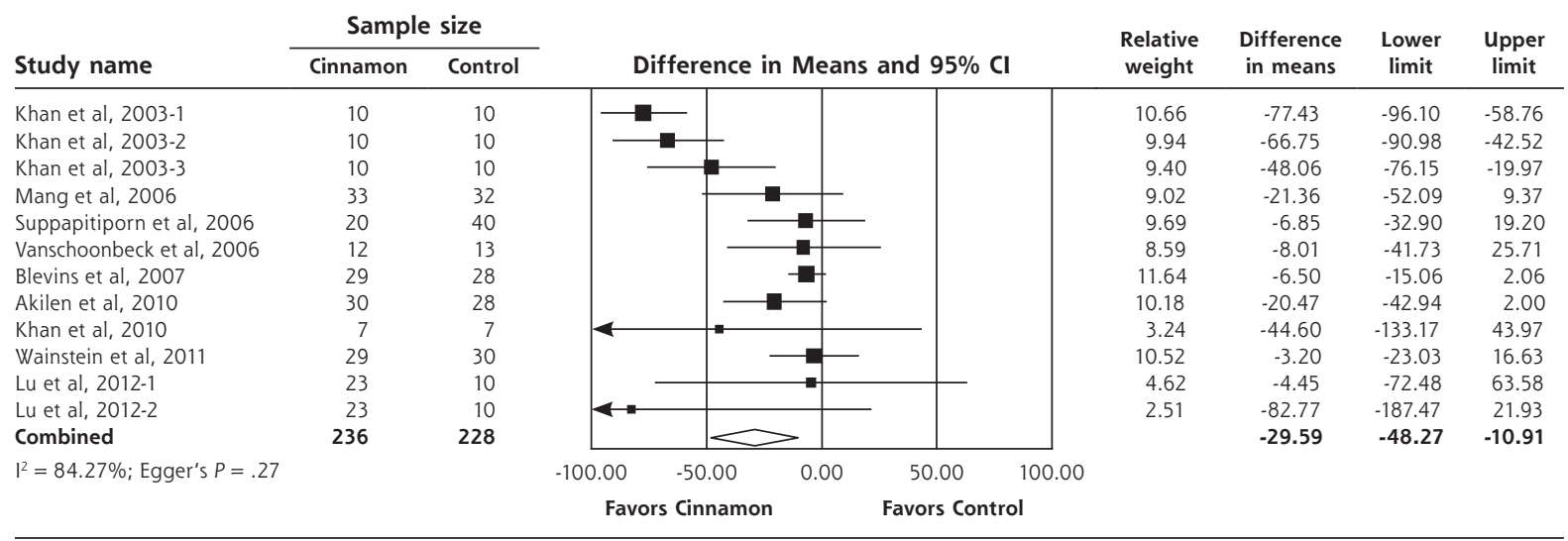

$\mathrm{HDL}=$ high-density lipoprotein; $\mathrm{LDL}=$ low-density lipoprotein.

Note: Squares represent individual studies, and size of the square represents the weight given to each study in the meta-analysis. Error bars represent $95 \%$ confidence intervals. The diamonds represent the pooled results. The solid vertical line extending upward from 0.00 is the null value.

comparatively lower doses of cinnamon $(1$ to $2 \mathrm{~g} / \mathrm{d}$ ) and included patients with baseline hemoglobin $\mathrm{A}_{1 \mathrm{c}}$ levels greater than $8 \%$ who were using concomitant hypoglycemic medications..$^{11,12}$

To put the results of our meta-analysis into better context, the reductions found can be compared with established therapies. As an example, the reductions in fasting plasma glucose levels $(-24.59 \mathrm{mg} / \mathrm{dL})$ are less than those achieved by metformin monotherapy $(-58 \mathrm{mg} / \mathrm{dL})^{22}$ and somewhat more than the newer oral agents, such as sitagliptin $(-16$ to $-21 \mathrm{mg} / \mathrm{dL}) .{ }^{23}$ Our results showed cinnamon to reduce LDL-C and triglyceride levels by approximately 9.4 and $29.6 \mathrm{mg} / \mathrm{dL}$, respectively. As a comparison, the lipid-lowering drugs pravastatin and gemfibrozil reduce LDL-C and triglyceride levels by approximately $50 \mathrm{mg} / \mathrm{dL}(26 \%)$ and 50 mg/dL (31\%), respectively. ${ }^{24,25}$

Results of the cumulative meta-analysis on hemoglobin $A_{1 c}$ levels showed that with each addition of a published RCT, there was no time point at which there was ever a statistically significant benefit. For the fasting plasma glucose outcome, cumulative meta-analysis showed that there was a statistically significant benefit at all time points and that the results have been stable since the publication of the RCT by Blevins and colleagues in $2007,{ }^{10}$ with subsequently published studies having little impact on the overall conclusion, although the treatment effect size is decreasing with time (Supplemental Figure 2).

This meta-analysis updates our earlier work published in 2008, which analyzed patients with type 1 or type 2 diabetes, and did not show any statistically significant effects on hemoglobin $\mathrm{A}_{1 \mathrm{c}}$, fasting plasma glucose, or lipid parameters. ${ }^{16}$ The inability to detect statistically significant results may be due to the limited number of trials available at the time. Our current findings align with the results found in our prior meta-analysis in terms of trend and magnitude. 
The addition of RCTs have increased the statistical power of the analysis and enabled us to find statistically significant results when using the same statistical methodology. Another meta-analysis was published by Davis and Yokoyama, who conducted a search through May 2010 and included trials in patients with type 2 diabetes, prediabetes and metabolic syndrome, impaired fasting glucose, and healthy volunteers. ${ }^{26}$ They evaluated only fasting plasma glucose levels and found a statistically significant reduction $(-8.77 \pm 3.52$ $\mathrm{mg} / \mathrm{dL}, P=.025)$ when using cinnamon compared with control. ${ }^{26}$ The results by Davis and Yokoyama likely showed more modest effects on fasting plasma glucose as the result of including patients without diagnosed diabetes.

Study characteristics of the trials included in the analysis may have contributed to clinical and statistical heterogeneity and are a limitation of this meta-analysis. Although all patients had type 2 diabetes, the populations studied varied among postmenopausal women, otherwise healthy adults, adults with comorbidities, such as hyperlipidemia, overweight, or obesity, and the use of concurrent medications. The range of cinnamon dose and dosage form may also contribute to heterogeneous results. In future investigations, a multivariate analysis controlling for multiple influential factors, such as dosage form, dose, and baseline lipid levels, may be necessary to identify the ideal product and population to exert benefit. The short duration of treatment may have limited the ability to see any changes in hemoglobin $A_{1 c}$ levels, because the duration ranged from 4 to 18 weeks. Hemoglobin $A_{1 c}$ levels are a reflection of a 3 -month average of glucose levels, and most trials were at least 3 months, so some effect should have been observed if it truly existed.

The results of this analysis may also be affected by the inconsistent quality of botanical research. The quantity of active cinnamaldehyde may vary among species and even among formulations. ${ }^{27}$ Consequently, it may be difficult to achieve predictable results if administering a cinnamon product to a patient. Additionally, variations in manufacturing processes may affect the quantity of active ingredient found in a specific formulation, as herbal products do not generally undergo the same manufacturing oversight as pharmacological products. ${ }^{28}$

Side effects of cinnamon have been poorly documented in humans, because most research focusing on safety and efficacy has been conducted either in vitro or in animals. Potential side effects found in animal studies include hepatotoxicity that results from coumarin isolates found in C cassia bark, decreased platelet counts, increased the risk of bleeding, decreased serum total cholesterol and triglyceride concentra- tions, and markedly increased HDL-C levels and allergy/hypersensitivity to cinnamon. ${ }^{2}$ These possible effects may be a concern for patients with impaired liver function, concurrent anticoagulant or antiplatelet therapy, patients on antilipidemic agents, and those who are known to be hypersensitive to cinnamon or its components. There are reports of nonimmunologic contact urticaria occurring in people coming into contact with cinnamon products ${ }^{3}$; however, because no human studies have been conducted to test this theory, these concerns remain hypothetical. Although our meta-analysis did not address safety data because the reporting of adverse events and tolerability were not consistent among the RCTs, all the included trials stated that no significant side effects were seen with cinnamon use.

With all meta-analyses, there is risk of publication bias. Our results suggested a high likelihood for publication bias on fasting plasma glucose, perhaps as a result of analyzing the results Khan and colleagues ${ }^{6}$ as 3 separate comparisons, which could result in an exaggerated lower effect size. Also the results from Khan et $a l^{6}$ could have affected the assessment for publication bias negatively because of the low sample size and large standard deviation in their study population. It is necessary to conduct trials in a long-term extension of cinnamon supplementation to better understand and investigate safety and tolerability of various oral formulations. With the small effect size seen on the analysis of hemoglobin $\mathrm{A}_{1 \mathrm{c}}$ levels, an unrealistically large sample size would be necessary to observe a statistically significant effect. It is unlikely that the addition of future RCTs will yield statistically significant results on hemoglobin $\mathrm{A}_{\mathrm{c}}$, and if they do, the results would unlikely be clinically significant. Further evaluation of fasting plasma glucose would also be unlikely to influence the statistical significance found, although it may modify the magnitude of effect seen.

Based on currently available literature, cinnamon may have a beneficial effect on fasting plasma glucose, LDL-C, HDL-C, and triglyceride levels in patients with type 2 diabetes. There is no statistically significant effect on hemoglobin $\mathrm{A}_{\mathrm{c},}$, however. Caution should be exercised in applying the results of this analysis to patient care because of the certainty of the dose and duration of cinnamon use and uncertainty of the ideal patient population.

To read or post commentaries in response to this article, see it online at http://www.annfammed.org/content/11/5/452.

Key words: cinnamon; type 2 diabetes mellitus; lipids; meta-analysis; review, systematic

Submitted June 25, 2012; submitted, revised, October 17, accepted November 11, 2012. 


\section{References}

1. Selvin E, Marinopoulos S, Berkenblit G, et al. Meta-analysis: glycosylated hemoglobin and cardiovascular disease in diabetes mellitus. Ann Intern Med. 2004;141(6):421-431.

2. Bantle JP, Wylie-Rosett J, Albright AL, et al; American Diabetes Association. Nutrition recommendations and interventions for diabetes: a position statement of the American Diabetes Association. Diabetes Care. 2008;31(Suppl 1):S61-S78.

3. Ulbricht C, Seamon E, Windsor RC, et al. An evidence-based systematic review of cinnamon (Cinnamomum spp.) by the Natural Standard Research Collaboration. J Diet Suppl. 2011;8(4):378-454.

4. Sheng X, Zhang Y, Gong Z, Huang C, Zang Y. Improved insulin resistance and lipid metabolism by cinnamon extract through activation of peroxisome proliferator-activated receptors. PPAR Res. 2008;581348.

5. Anand P, Murali KY, Tandon V, Murthy PS, Chandra R. Insulinotropic effect of cinnamaldehyde on transcriptional regulation of pyruvate kinase, phosphoenolpyruvate carboxykinase, and GLUT4 translocation in experimental diabetic rats. Chem Biol Interact. 2010;186(1):72-81.

6. Khan A, Safdar M, Ali Khan MM, Khattak KN, Anderson RA. Cinnamon improves glucose and lipids of people with type 2 diabetes. Diabetes Care. 2003;26(12):3215-3218.

7. Mang $B$, Wolters $M$, Schmitt $B$, et al. Effects of a cinnamon extract on plasma glucose, $\mathrm{HbA}$, and serum lipids in diabetes mellitus type 2. Eur J Clin Invest. 2006;36(5):340-344.

8. Suppapitiporn S, Kanpaksi N, Suppapitiporn S. The effect of cinnamon cassia powder in type 2 diabetes mellitus. J Med Assoc Thai. 2006;89(Suppl 3):S200-S205.

9. Vanschoonbeek K, Thomassen BJ, Senden JM, Wodzig WK, van Loon LJ. Cinnamon supplementation does not improve glycemic control in postmenopausal type 2 diabetes patients. J Nutr. 2006; 136(4):977-980.

10. Blevins SM, Leyva MJ, Brown J, Wright J, Scofield RH, Aston CE. Effect of cinnamon on glucose and lipid levels in non insulin-dependent type 2 diabetes. Diabetes Care. 2007;30(9):2236-2237.

11. Crawford P. Effectiveness of cinnamon for lowering hemoglobin A1C in patients with type 2 diabetes: a randomized, controlled trial. J Am Board Fam Med. 2009;22(5):507-512.

12. Akilen R, Tsiami A, Devendra D, Robinson N. Glycated haemoglobin and blood pressure-lowering effect of cinnamon in multi-ethnic Type 2 diabetic patients in the UK: a randomized, placebo-controlled, double-blind clinical trial. Diabet Med. 2010;27(10):1159-1167.

13. Khan R, Khan Z, Shah S. Cinnamon may reduce glucose, lipid and cholesterol level in type 2 diabetic individuals. Pakistan J Nutr. 2010; 9(5):430-433.

14. Wainstein J, Stern N, Heller S, Boaz M. Dietary cinnamon supplementation and changes in systolic blood pressure in subjects with type 2 diabetes. J Med Food. 2011;14(12):1505-1510.
15. Lu T, Sheng H, Wu J, Cheng Y, Zhu J, Chen Y. Cinnamon extract improves fasting blood glucose and glycosylated hemoglobin level in Chinese patients with type 2 diabetes. Nutr Res. 2012;32(6):408-412.

16. Baker WL, Gutierrez-Williams G, White CM, Kluger J, Coleman Cl. Effect of cinnamon on glucose control and lipid parameters. Diabetes Care. 2008;31(1):41-43.

17. Higgins JP, Altman DG, Gøtzsche PC, et al; Cochrane Bias Methods Group; Cochrane Statistical Methods Group. The Cochrane Collaboration's tool for assessing risk of bias in randomised trials. BMJ. 2011;343:d5928.

18. DerSimonian R, Laird N. Meta-analysis in clinical trials. Control Clin Trials. 1986;7(3):177-188.

19. Follmann D, Elliott P, Suh I, Cutler J. Variance imputation for overviews of clinical trials with continuous response. J Clin Epidemiol. 1992;45(7):769-773.

20. Higgins JP, Thompson SG, Deeks JJ, Altman DG. Measuring inconsistency in meta-analyses. BMJ. 2003;327(7414):557-560.

21. Egger M, Davey Smith G, Schneider M, Minder C. Bias in metaanalysis detected by a simple, graphical test. BMJ. 1997;315(7109): 629-634.

22. Inzucchi SE, Maggs DG, Spollett GR, et al. Efficacy and metabolic effects of metformin and troglitazone in type II diabetes mellitus. N Engl J Med. 1998;338(13):867-872.

23. Aschner P, Kipnes MS, Lunceford JK, Sanchez M, Mickel C, Williams-Herman DE; Sitagliptin Study 021 Group. Effect of the dipeptidyl peptidase-4 inhibitor sitagliptin as monotherapy on glycemic control in patients with type 2 diabetes. Diabetes Care. 2006;29(12): 2632-2637.

24. Influence of pravastatin and plasma lipids on clinical events in the West of Scotland Coronary Prevention Study (WOSCOPS). Circulation. 1998;97(15):1440-1445.

25. Rubins HB, Robins SJ, Collins D, et al; Veterans Affairs High-Density Lipoprotein Cholesterol Intervention Trial Study Group. Gemfibrozil for the secondary prevention of coronary heart disease in men with low levels of high-density lipoprotein cholesterol. $N$ Engl J Med. 1999;341(6):410-418.

26. Davis PA, Yokoyama W. Cinnamon intake lowers fasting blood glucose: meta-analysis. J Med Food. 2011;14(9):884-889.

27. Corns CM. Herbal remedies and clinical biochemistry. Ann Clin Biochem. 2003;40(Pt 5):489-507.

28. Guidance for industry: botanical drug products. U.S. Department of Health and Human Services. Food and Drug Administration. Center for Drug Evaluation and Research. http://www.fda.gov/downloads/Drugs/GuidanceComplianceRegulatoryInformation/Guidances/ ucm070491.pdf. Accessed Oct 12th, 2012. 